\title{
Effect of Bulgarian Split Squat Exercise on 20 Meters Sprint Performance
}

\author{
Nurten Dinç ${ }^{1}$, Ümit Hayta ${ }^{1}$ \\ ${ }^{1}$ Manisa Celal Bayar University, Faculty of Sports Sciences, Turkey \\ Correspondence: Nurten Dinç, Manisa Celal Bayar University, Faculty of Sports Sciences, Turkey.
}

Received: June 26, 2018

Accepted: July 24, $2018 \quad$ Online Published: August 6, 2018

doi:10.11114/jets.v6i9.3391

URL: https://doi.org/10.11114/jets.v6i9.3391

\begin{abstract}
Introduction and Aim: The results of the research show that the results of squat exercise affect the sprint performance and the results are different. For this reason, our aim in the study is to investigate the effect of Bulgarian split squat exercise, which is a squat type, on the sprint performance of 20 meters.

Method: 10 sedanters who had a mean age of $20 \pm 198$ years, a mean height of 175,4 $\pm 5,35$, a mean weight of $66,42 \pm$ 7,68 and a BMI of 21,52 \pm 1,37, from Manisa Celal Bayar University Faculty of Sports Sciences voluntarily participated in the study. Given the exercise protocol, participants were warmed up by low tempo jogging for 5 minutes. After the rests, participants participated in Bulgarian squat exercises in the form of 5-8 repetitions at 60-80\% of $1 \mathrm{RM}$. After each Bulgarian split squat, a 4-minute rest was given and then two 20-meter sprint tests were performed (STAE). After each sprint was given a 3-minute rest. The same group performed two 20-meter sprint tests 72 hours later without Bulgarian split squat exercise (STWE). The best score was taken from two sprint tests. The sprint performance after exercise was compared with the sprint performance without exercise. The data were analysed using the repeated measures method in the SPSS program.

Findings and Conclusion: No significant difference was found $(\mathrm{p}>0.05)$ between the exercise types when the data obtained were compared. The reason for this is thought to be the fact that the subjects participating in the study were sedentary and in addition, the exercise caused fatigue on the subjects.
\end{abstract}

Keywords: sprint performance, postactivation potentation, bulgarian split squat

\section{Introduction}

The sprint run consists of 5 parts including start, acceleration, maximum velocity, deceleration and finish. The main aim is the ballistic cyclic movements that are exhibited in the sprint conditions. In these cyclical movements, muscles accelerate or decelerate parts of the body by straining, relaxing or stretching. Thus, muscles move parts of the body in different ways with different widths by determining the force to be applied ideally and balancing diarthroses, absorbing shocks and amortising the effects with external factors (Kale et al., 2008). Also, since the sprint run is one of the fastest movements that a person can make from natural directions, it is necessary to determine, from biomechanical and physiological perspective, what sort of forces and angles are required in order to reach which muscles strain earlier and to reach high performance (Nesser et al., 1996).

Because of the advantage of having higher speeds, sprint training has focused on research on improving sprint performance using countless training methods including sprint drills, resistance to accelerations, weight training, combined resistance, speed training and plyometrics (De Villareal et al.,2012; Rimmer \& Sleiyvert, 2000). Sprint running performance is considered as a key component of success in a variety of competitive sports or multiple sprint sports (football, rugby, and hockey) (Highton et al 2012). Sprint needs strength and acceleration at high levels to defeat the motionlessness of the body mass. A number of studies have investigated the relationship between power and sprint performance and have shown that stronger athletes generally perform better during sprint demonstration (Mcbride et al., 2009; Wisloff et al., 2004). This can be explained by the fact that the reaction forces and the impulse are powerful determinants of sprint performance (Comfort et al., 2012; Hunter et al., 2005).

While studies investigate the relationship between power and speed performance, several methods including isokinetic, machine squat and free-weight squat, have been used to evaluate the strength. The strongest correlations were obtained with free-weight squat (Comfort et al., 2012). It is seen that there is a clear relationship between maximal free weight squat power and sprint performance in trained individuals. A strong correlation has been found between one repletion 
maximum (1RM) squat performance and a sprint time of 10 meters (Wisloff et al., 2004). Recent studies have reported that explosive jumping performance increases after weighted workout in a short time with high intensity warm-up (non-contrast or non-complex training) (Chiu et al., 2003).

This temporary increase in muscle performance has been attributed to a phenomenon known as post-activation potention (PAP), a condition in which the emergence of acute muscle strength is strengthened as a consequence of the contractile past (Sale, 2002). Although most of the PAP studies have been based on jumping, only a few studies have investigated the effects on sport-specific performance (Mcbride et al., 2005; Paff, 1997). These studies have been reported to improve dynamic performance in competitive sports such as cycling and sprinting (Chatzopoulos et al., 2007; Hamada et al., 2000; Linder et al., 2010; Mcbride et al., 2005). In a study of Chatzopoulos et al. (2007) conducted, it was reported that for amateurs, $90 \%$ of $1 \mathrm{TM}$ increased the effect of PAP on short distances of 10 and 30 meters after 10-time repetitions of single foot squat. Although Pfaff's (1997) study has reported no numerical values, a significant increase in the performance of elite sprinters performing a warm-up with heavy resistance which constitutes $90 \%$ of 1 RM for 5 sets with 1-time repetitions in back squat exercise has been detected. In this study, we aim to investigate the effect of Bulgarian split squat exercise on the 20-meter sprint performance.

\section{Method}

This study was designed to investigate the effect of Bulgarian split squat exercise on the 20-meter sprint performance. When compared with squat exercise, it is considered that split squat exercises have a positive effect on sprint performance because of differences in the angles. Participants in the study participated in two sessions. In the first session, a sprint test was performed after performing Bulgarian split squat exercise. In the second session, after 72 hours from the first session, a sprint test was performed without performing a Bulgarian split squat exercise.

\subsection{Subjects}

Ten volunteers $(\mathrm{N}=10)$ from Manisa Celal Bayar University, Faculty of Sport Sciences, who had not been trained, participated in this study. None of the participants had a history of hypertension, coronary heart disease, anemia and diabetes. Participants' anthropometric measurements (bio-space, South Korea) were measured using an inbody 230 multi-frequency bioelectrical impedance device. With this measurement, participant's weight, body mass index, body fat ratio, lean muscle mass measurements were taken. In the linear measurement of the participations, mst-mkii mechanical height scale was used. Before participating in the test, participants were asked to submit their health reports. All the participants completed a medical history inventory form and signed the informed consent form that explained the objectives and risks of the study. The ethical committee approval of the study was taken from the ethics committee of Health Sciences of Manisa Celal Bayar University, Faculty of Medicine.

\subsection{Test Protocol}

Participants performed warm-up with low tempo jogging for 5 minutes. In order to see the current status of the participants, a work-out with one set with 8 repetitions was conducted and 4-minute rests were allowed. After having a rest, the participants did a Bulgarian split squat exercise in the form of 5-8 repetitions at \%60-80 of 1RM. After the Bulgarian split squat, a four-minute-rest was given and then two 20-meter sprint tests were performed. A three-minute-rest was given to them after each sprint. The same group performed two 20-meter sprint tests 72 hours later without performing a Bulgarian split squat exercise in the second session.

\subsubsection{One Repetition Maximum Estimation and Bulgarian Split Squat Exercise}

The participant was first introduced to the exercise technique. Participants who had an appropriate technique performed the actual test protocol. 1RM was recorded as the maximum resistance that can be lifted once with complete movement (Faigenbaum et al., 2003). Bulgarian split squat was calculated using the following equation to determine the maximal weight of the exercise (maximum 1 repetition $=$ weight $/(1.0278-(, 0278 *$ repetition)) (Brzycki, 1993). After a maximum of 1 repetition was determined, the subjects performed a total of 2 sets of Bulgarian split squat exercises, with 5 repetitions and 8 repetitions at a maximum of $60-80 \%$. A rest period of 4 minutes was provided between the sets.

\subsubsection{0 meters Sprint Test after Bulgarian Split Squat}

Participants showed sprint performance in a rubber covered area after the Bulgarian split squat exercise. Participants' sprint duration (Brower Timing Systems, Draper, UT) was recorded using a photocell.

\subsection{Statistical Analyses}

The SPSS 22 package program was used to perform the statistical analysis of the study. The difference between the participants' sprint performance by doing the Bulgarian split squat exercise and the exercise by not doing the Bulgarian split exercise was determined with intra-group analysis of Wilcoxon signed rank test. The significance limit used during the study was $\mathrm{P}<0.05$. 


\section{Results}

According to Wilcoxon signed rank test; in this study there is no significant between STAE and STWE (P>0.05). Table 1 shows the demographic information of subjects participating in the study. Respectively, it is stated that age is $20 \pm$ 1.98 years, height is $175.4 \pm 5.35 \mathrm{~cm}$., weight is $66.42 \pm 7.68 \mathrm{~kg}$., BMI is $21.52 \pm 1.37$, STAE is $3.21 \pm 0.115$ seconds, STWE is $3.16 \pm 0.133$ seconds.

Table 1. Demographic Information of Participants

\begin{tabular}{lllllll}
\hline $\mathrm{N}$ & Age (Year) & Height $(\mathrm{cm})$ & Kilos $(\mathrm{kg})$ & BMI(SI) & STAE $(\mathrm{sec})$ & STWE $(\mathrm{sec})$ \\
\hline 10 & $20 \pm .1 .98$ & $175.4 \pm 5.35$ & $66.42 \pm 7.68$ & $21.52 \pm 1.37$ & $3.21 \pm .115$ & $3.16 \pm .133$ \\
\hline
\end{tabular}

STAE: Sprint Test After Exercise. STWE: Sprint Test Without Exercise. BMI: Body Mass Index

In the Table 2 the difference between the participants' sprint performance with the Bulgarian split squat exercise (STAE) and the sprint performances without the Bulgarian split squat exercise (STWE) is given. According to Table 2, data of sprint test without exercise and sprint test after exercise have been analysed by Wilcoxon-marked Rank Test and there has been no statistically significant difference between them $\mathrm{P}>0.05$.

Table 2. Analysis of Participants Sprint Data

\begin{tabular}{|c|c|c|c|c|c|}
\hline & & $N$ & ORT\&S.S & $Z$ & $P$ \\
\hline \multirow{2}{*}{ SPRINT } & STAE & 10 & $3.2 \pm 115$ & \multirow{2}{*}{-1.479} & \multirow{2}{*}{0.139} \\
\hline & & 10 & $3.1 \pm 133$ & & \\
\hline
\end{tabular}

STAE: Sprint Test after Exercise. STWE: Sprint Test without Exercise P< 0.05

\section{Discussion}

In this study investigating the effect of the Bulgarian split squat exercise on the 20-meter sprint performance, there was no statistically significant difference between the sprint performance and the Bulgarian split squat exercise. In the literature, the study has indicated a weak and insignificant correlation between squat with Smith machine and 10-and-40-meter sprint times in athletes' work (Harris et al., 2008). In addition, Till and Cook (2009) state in their study that there is no significant difference in sprint and vertical jump of potential practices after activity. Lim \& Kong (2013), report in the study performed in 4 different days that there is no improvement in short-range sprint performance after PAP protocols and four-minute-rest period with single-articulated or multi-articulated irrespective of isometric or dynamics. Vanderka et al. (2016), show in their study that there is no significant difference in the sprint time for athletes and footballers after jump squat, and the sprint time is negatively affected. On the other hand, Crewther et al. (2011), report that there is no significant difference on rugby players in sprint performance after a single squat set with three repetitions exercise of $90 \%$ of $1 \mathrm{RM}$. Since these studies are parallel to our study, the tests are performed on elite athletes. This study has come up with different results since the subjects are sedentary.

On the other hand, there are studies that show similar significant increases in sprint performances apart from jumping performance and they differ from our study. In a study, it is stated that there is a strong correlation between 1RM squat performance and 10-meter sprint time of trained subjects (Wisloff et al., 2004). While Chatzopolous at al. (2007) have found a $2.36 \%$ improvement in 10-meter sprint performance and a $1.77 \%$ improvement in 30 -meter sprint performance after 10 single foot squats, Mcbride et al. (2005), have found in his work that there is a $0.87 \%$ increase in sprint performance after squat exercise (90\% in $1 \mathrm{RM}$ ). Wyland et al. (2015), report in the study involving a 5 -set-with-3-repetition exercise with the $\% 85$ of $1 \mathrm{RM}$ that there is a significant improvement in sprint timing in a protocol involving heavy-load squat exercise 4 minutes earlier. In a study conducted, it is stated that there is a significant difference between the best sprint timing and sprint average values at the protocol performed in the forms of a one-set-five-repetition exercise with $\% 50$ of 1RM, a three-repetition exercise with $\% 70$ of 1RM, and a five-set-one-repetition exercise with \%90 of 1RM (Okuno et al., 2013). Unlike the above studies, Yetter \& Moir (2008), determine that the front squat does not increase the sprint performance but the back squat provided faster sprint speeds in 10-20 $\mathrm{m}$ and 30-40 $\mathrm{m}$ while it does not provide this in 0-10 $\mathrm{m}$ and 20-30 m. Besides, these studies do not support our study; it is also different from our study in terms of the participants who are not elite athletes.

In this study, it is thought that the reason why there is no significant difference between sprint performance after Bulgarian split squat exercise and sprint performance without this exercise is because the participations are sedentary and they also get tired after the exercise. It is recommended that different studies which intent to investigate the effect of the split squat exercise on the $20 \mathrm{~m}$ sprint performance should take participants' athletic backgrounds and resting time into consideration. 


\section{Practical Applications}

The findings of the current study indicate that the Bulgarian split squat exercise should be done by trained athletes rather than sedanters. This type of exercise is found to have a positive impact on the sprint time of the athletes. So, sprinters or athletes in need of sprint while doing sports may take the advantage of doing Bulgarian split squat exercise right before the competitions. The protocol used in this study is likely to come up with different results among elite athletes depending on their sports background. Besides, trainers and researchers can develop and use this protocol in different ways such as shortening or extending the rest time, employing various exercise types, and arranging the intensity of the exercises.

\section{References}

Andersen, V., Fimland, M. S., Brennset, Ø., Haslestad, L. R., Lundteigen, M. S., Skalleberg, K., \& Saeterbakken, A. H., (2014). Muscle activation and strength in squat and bulgarian squat on stable and unstable surface. International Journal of Sports Medicine, 35(14), 1196-1202. https://doi.org/10.1055/s-0034-1382016

Brzycki, M. (1993). Strength testing - predicting a one-rep max from reps-to-fatigue. Journal of Physical Education, Recreation \& Dance, 64(1), 88-90. https://doi.org/10.1080/07303084.1993.10606684

Chatzopoulos, D. E., Michailidis, C. J., Giannakos, A. K., Alexiou, K. C., Patikas, D. A., Antonopoulos, C. B., \& Kotzamanidis, C. M. (2007). Postactivation potentiation effects after heavy resistance exercise on running speed. The Journal of Strength \& Conditioning Research, 21(4), 1278-1281. https://search.proquest.com/openview/2ad99a65952bb3988f6a8442050df52e/1?pq-origsite=gscholar\&cbl=30912

Chiu, L. Z., Fry, A. C., Weiss, L. W., Schilling, B. K., Brown, L. E., \& Smith, S. L. (2003). Postactivation potentiation response in athletic and recreationally trained individuals. The Journal of Strength \& Conditioning Research, 17(4), 671-677.

http://www.academia.edu/download/42713882/Postactivation_Potentiation_Response_in_20160215-27115-1r3caq 6.pdf

Comfort, P., Bullock, N., \& Pearson, S. J. (2012). A comparison of maximal squat strength and 5-, 10-, and 20-meter sprint times, in athletes and recreationally trained men. The Journal of Strength \& Conditioning Research, 26(4), 937-940. https://doi.org/10.1519/JSC.0b013e31822e5889

Crewther, B. T., Kilduff, L. P., Cook, C. J., Middleton, M. K., Bunce, P. J., \& Yang, G. Z. (2011). The acute potentiating effects of back squats on athlete performance. The Journal of Strength \& Conditioning Research, 25(12), 3319-3325. https://doi.org/10.1519/JSC.0b013e318215f560

De Villarreal, E. S., Requena, B., \& Cronin, J. B. (2012). The effects of plyometric training on sprint performance: a meta-analysis. The Journal of Strength \& Conditioning Research, 26(2), https://doi.org/10.1519/JSC.0b013e318196b7c6

Faigenbaum, A. D., Milliken, L. A., \& Westcott, W. L. (2003). Maximal strength testing in healthy children. The Journal of Strength \& Conditioning Research, 17(1), 162-166.

https://pdfs.semanticscholar.org/d8de/7bdbab794e654a71ffafc85253850f1e388b.pdf

Hamada, T. A. K. U., Sale, D. G., \& Macdougall, J. D. (2000). Postactivation potentiation in endurance-trained male athletes. Medicine and Science in Sports and Exercise, 32(2), 403-411. https://doi.org/10.1097/00005768-200002000-00022

Harris, N. K., Cronin, J. B., Hopkins, W. G., \& Hansen, K. T. (2008). Relationship between sprint times and the strength/power outputs of a machine squat jump. The Journal of Strength \& Conditioning Research, 22(3), 691-698. https://doi.org/10.1519/JSC.0b013e31816d8d80

Highton, J. M., Lamb, K. L., Twist, C., \& Nicholas, C. (2012). The reliability and validity of short-distance sprint performance assessed on a nonmotorized treadmill. The Journal of Strength \& Conditioning Research, 26(2), 458-465. https://doi.org/10.1519/JSC.0b013e318225f384

Hunter, J. P., Marshall, R. N., \& McNair, P. J. (2005). Relationships between ground reaction force impulse and kinematics of sprint-running acceleration. Journal of Applied Biomechanics, 21(1), 31-43. https://doi.org/10.1123/jab.21.1.31

Kale, M., A., Bayrak, C., \& Acikada, C. (2008) Effect of competition training to acceleration kinematics and physiological variables in sprinters, Journal of Sport Sciences., 19 (1), 35-53. 
Lim, J. J., \& Kong, P. W. (2013). Effects of isometric and dynamic postactivation potentiation protocols on maximal sprint performance. The Journal of Strength \& Conditioning Research, 27(10), 2730-2736. https://doi.org/10.1519/JSC.0b013e3182815995

Linder, E. E., Prins, J. H., Murata, N. M., Derenne, C., Morgan, C. F., \& Solomon, J. R. (2010). Effects of preload 4 repetition maximum on 100-m sprint times in collegiate women. The Journal of Strength \& Conditioning Research, 24(5), 1184-1190. https://doi.org/10.1519/JSC.0b013e3181d75806

McBride' J. M., Blow, D., Kirby, T. J., Haines, T. L., Dayne, A. M., \& Triplett, N. T. (2009). Relationship between maximal squat strength and five, ten, and forty yard sprint times. The Journal of Strength \& Conditioning Research, 23(6), 1633-1636. https://doi.org/10.1519/JSC.0b013e3181b2b8aa

Mcbride, J. M., Nimphius, S., \& Erickson, T. M. (2005). The acute effects of heavy-load squats and loaded countermovement jumps on sprint performance. The Journal of Strength \& Conditioning Research, 19(4), 893-897. https://search.proquest.com/openview/f84dc5bf9cef3a3acc4c1a1be595d427/1?pq-origsite=gscholar\&cbl=30912

Nesser, T. W., Latin, R. W., Berg, K., \& Prentice, E. (1996). Physiological determinants of 40-meter sprint performance in young male athletes. Journal of Strength and Conditioning Research, 10, 263-267.

Okuno, N. M., Tricoli, V., Silva, S. B., Bertuzzi, R., Moreira, A., \& Kiss, M. A. (2013). Post activation potentiation on repeated-sprint ability in elite handball players. The Journal of Strength \& Conditioning Research, 27(3), 662-668. https://doi.org/10.1519/JSC.0b013e31825bb582

Pfaff, D. (1997). Training methods for elite sprinters. In Appalachian High Performance Conference. Boone, NC.

Rimmer, E., \& Sleivert, G. (2000). Effects of a Plyometrics Intervention Program on Sprint Performance. The Journal of Strength \& Conditioning Research, 14(3), 295-301.

http://www.neosportsplant.com/Performance/Articles/Plyometrics/Effects_of_a_Plyometrics_Intervention_Progra m_on.9.pdf

Sale, D. G. (2002). Postactivation potantion: role in human performance. Exercise and Sport Sciences Reviews, 30(3), 138-143. https://doi.org/10.1097/00003677-200207000-00008

Till, K. A., \& Cooke, C. (2009). The effects of postactivation potentiation on sprint and jump performance of male academy soccer players. The Journal of Strength \& Conditioning Research,23(7), 1960-1967. https://doi.org/10.1519/JSC.0b013e3181b8666e

Vanderka, M., Krcmár, M., Longová, K., \& Walker, S. (2016). Acute effects of loaded half-squat jumps on sprint running speed in track and field athletes and soccer players. The Journal of Strength \& Conditioning Research, 30(6), 1540-1546. https://doi.org/10.1519/JSC.0000000000001259

Wisløff, U., Castagna, C., Helgerud, J., Jones, R., \& Hoff, J. (2004). Strong correlation of maximal squat strength with sprint performance and vertical jump height in elite soccer players. British journal of sports medicine, 38(3), 285-288. http://dx.doi.org/10.1136/bjsm.2002.002071.

Wyland, T. P., Van Dorin, J. D., \& Reyes, G. F. C. (2015). Postactivation potentation effects from accommodating resistance combined with heavy back squats on short sprint performance. The Journal of Strength \& Conditioning Research, 29(11), 3115-3123. https://doi.org/10.1519/JSC.0000000000000991

Yetter, M., \& Moir, G. L. (2008). The acute effects of heavy back and front squats on speed during forty-meter sprint trials. The Journal of Strength \& Conditioning Research, 22(1), 159-165.

https://doi.org/10.1519/JSC.0b013e31815f958d

\section{Copyrights}

Copyright for this article is retained by the author(s), with first publication rights granted to the journal.

This is an open-access article distributed under the terms and conditions of the Creative Commons Attribution license which permits unrestricted use, distribution, and reproduction in any medium, provided the original work is properly cited. 